\title{
Stat3-Syk Molecular Complex as A Target for Anti-Cancer Nanomedicines
}

\section{Fatih M Uckun ${ }^{1,2,3 *}$}

${ }^{1}$ Keck Medical Center of University of Southern California, Los Angeles, CA

${ }^{2}$ Children's Center for Cancer and Blood Diseases, Children's Hospital Los Angeles, Los Angeles, CA

${ }^{3}$ The Saban Research Institute, Children's Hospital Los Angeles, Los Angeles, CA

Spleen Tyrosine Kinase (SYK) can activate the oncogenic transcription factor "The Signal Transducer and Activator of Transcription 3" (STAT3) to induce expression of STAT3 target genes that protect cancer cells against chemotherapy and ionizing radiation [1]. A meta-analysis using the Oncomine database revealed a marked enrichment of the most discriminating SYK-dependent anti-apoptotic genes in human leukemias and lymphomas [2]. In patients that showed upregulation of SYK, the majority of comparisons also showed significant upregulation of the STAT3 target genes. SYK has also been shown to regulate the activation of the mammalian target of rapamycin (mTOR), an evolutionarily conserved serine/threonine kinase that is controlled by the SYK-regulated PI3-K/AKT and MAPK/ERK pathways $[2,3]$. Notably, the anti-apoptotic NFKB is also regulated by SYK [2]. Thus, STAT3-SYK molecular complex is a master regulator of apoptosis and neoplastic expansion in leukemias and lymphomas [2]. Therefore, rationally designed inhibitors of this molecular target may provide the foundation for therapeutic innovation against leukemias and lymphomas. Several structurally distinct and highly potent small molecule inhibitors of SYK tyrosine kinase ATP binding site have been developed [4-7]. In addition to small molecule tyrosine kinase inhibitors, antisense oligonucleotides and small interfering RNAs (siRNAs) have also been developed to knock down SYK expression $[8,9]$.

We recently discovered the pentapeptide mimic 1,4-Bis (9-O dihydroquinidinyl) phthalazine / hydroquinidine 1,4-phathalazinediyl diether (C-61) as a tyrosine kinase inhibitor targeting the substrate binding $\mathrm{P}$-site of SYK and as a novel drug candidate against B-lineage leukemias and lymphomas $[1,4,5]$. C-61 inhibits SYK in both cellfree and cellular kinase assays at nanomolar concentrations, acts as a potent inducer of apoptosis in chemotherapy-resistant primary leukemic B-cell precursors taken directly from relapsed B-lineage ALL patients, exhibits favorable pharmacokinetics in mice and non-human primates, and eradicates leukemia-initiating cells in SCID mouse xenograft models of chemotherapy-resistant human B-lineage ALL at dose levels non-toxic to mice and non-human primates [1,4,5]. Further development of C-61 may provide the foundation for therapeutic innovation against chemotherapy-resistant leukemias and lymphomas. C-61 prevented radiation-induced activation of SYK and markedly augmented radiation-induced apoptosis of leukemia/lymphoma cells and enhanced the anti-leukemic potency of total body irradiation (TBI) in the context of hematopoietic stem cell transplantation (SCT) [5]. Based on this preclinical proof-of-principle study, we hypothesize that the incorporation of C-61 into the pre-transplant TBI regimens of patients with recurrent or high risk leukemias/lymphomas will help overcome the radiochemotherapy resistance of their cancer cells and thereby improve their treatment response and survival outcome after hematopoietic SCT.

The efficient delivery of the various SYK inhibitors by leveraging nanotechnology holds particular promise. Liposomal nanoparticle therapeutics containing active anti-cancer agents, including targetspecific small molecules and siRNA, may provide the foundation for potentially more effective and less toxic anti-cancer treatment strategies due to their improved pharmacokinetics, reduced systemic toxicity, and increased intra-tumoral/intra-cellular delivery [10-14]. Nanoparticles have been coated with Polyethylene Glycol (PEG) in an attempt to render them resistant against protein adsorption, enhance their biocompatibility, and to stabilize them against agglomeration in biological environments. PEGylated nanoparticles with diameters around 100-nm may become long-circulating in the blood stream and have been called stealth particles since they can evade recognition by $\mathrm{T}$-cells and macrophages and avoid rapid clearance by the immune system [10-14]. Nanoparticles that are sterically stabilized by PEG polymers on their surface and have surface charges that are slightly negative or slightly positive have minimal self-self or self-nonself interactions and improved pharmacokinetics. PEGylation of nanoparticles creates a hydrophilic surface and leads to increased protein solubility, reduced immunogenicity, prolonged plasma half-life due to prevention of rapid renal clearance, and reduced clearance by the RES system due to decreased macrophage capture and opsonization [10-14]. The rationally engineered nanoparticle constructs of SYK inhibitors are likely to be less toxic and more effective than free molecules.

Nanoparticles can be functionalized with a tumor targeting moiety such as a ligand or (Single chain variable fragment) scFv directed against a surface receptor on cancer cells in order to achieve optimal tumor targeting and site-specific drug delivery to further reduce their toxicity and improve their efficacy [10-14]. When linked with tumor targeting moieties nanoparticles can reach cancer cells carrying the target receptors with high affinity and precision. The targeting ligands enable nanoparticles to bind to cell surface receptors and enter cells by receptor-mediated endocytosis. In our own program, efforts are underway to prepare nanoparticles targeted against the CD19 antigen on childhood leukemia cells. Likewise, we are preparing nanoparticles functionalized with recombinant human Insulin-Like Growth Factor 1 (IGF1) to target surface IGF1 receptors on solid tumor cells. Rationally engineered nanoparticle therapeutics containing C-61 may provide the foundation for potentially more effective and less toxic anti-cancer treatment strategies due to their improved pharmacokinetics, reduced systemic toxicity, and increased intra-tumoral/intra-cellular delivery [5]. We hypothesize that the nanotechnology-enabled delivery of C-61 [14] will be associated with reduced toxicity enhanced potency compared to the free drug delivery with the salt-formulation used in our recent preclinical studies. Further development of rationally

${ }^{*}$ Corresponding author: Fatih M. Uckun, Professor of Pediatrics, University of Southern California, Los Angeles, CA, E-mail: uckun@usc.edu

Received June 25, 2012; Accepted June 25, 2012; Published June 27, 2012

Citation: Uckun FM (2012) Stat3-Syk Molecular Complex as A Target for AntiCancer Nanomedicines. J Nanomed Nanotechol 3:e110. doi:10.4172/2157$7439.1000 \mathrm{e} 110$

Copyright: $\odot 2012$ Uckun FM. This is an open-access article distributed under the terms of the Creative Commons Attribution License, which permits unrestricted use, distribution, and reproduction in any medium, provided the original author and source are credited. 
Citation: Uckun FM (2012) Stat3-Syk Molecular Complex as A Target for Anti-Cancer Nanomedicines. J Nanomed Nanotechol 3:e110. doi:10.4172/2157-7439.1000e110

designed SYK inhibitors and their nanoscale formulations may provide the foundation for therapeutic innovation against a broad spectrum of cancers, especially leukemias and lymphomas.

\section{Acknowledgments}

F.M.U is supported by DHHS/NIH grants U01-CA-151837, R01-CA-154471, Couples against Leukemia Foundation and a V Foundation Translational Research Grant.

\section{References}

1. Uckun FM, Qazi S, Ma H, Tuel-Ahlgren L, Ozer Z (2010) STAT3 is a substrate of SYK tyrosine kinase in B-lineage leukemia/lymphoma cells exposed to oxidative stress. Proc Natl Acad Sci U S A 107: 2902-2907.

2. Uckun FM, Qazi S (2010) Spleen tyrosine kinase as a molecular target for treatment of leukemias and lymphomas. Expert Rev Anticancer Ther 10: 14071418.

3. Riccaboni M, Bianchi I, Petrillo P (2010) Spleen tyrosine kinases: biology, therapeutic targets and drugs. Drug Discov Today 15: 517-530.

4. Uckun FM, Ek RO, Jan ST, Chen CL, Qazi S (2010) Targeting SYK kinasedependent anti-apoptotic resistance pathway in B-lineage acute lymphoblastic leukaemia (ALL) cells with a potent SYK inhibitory pentapeptide mimic. $\mathrm{Br} J$ Haematol 149: 508-517.

5. Uckun FM, Dibirdik I, Qazi S (2010) Augmentation of the antileukemia potency of total-body irradiation (TBI) by a novel P-site inhibitor of spleen tyrosine kinase (SYK). Radiat Res 174: 526-31.
6. Liddle J, Atkinson FL, Barker MD, Carter PS, Curtis NR, et al. (2011) Discovery of GSK143, a highly potent, selective and orally efficacious spleen tyrosine kinase inhibitor. Bioorg Med Chem Lett 21: 6188-6194.

7. Hirabayashi A, Mukaiyama $H$, Kobayashi $H$, Shiohara $H$, Nakayama $S$, et al (2008) A novel Syk family kinase inhibitor: design, synthesis, and structureactivity relationship of 1,2,4-triazolo[4,3-c]pyrimidine and 1,2,4-triazolo[1,5-c] pyrimidine derivatives. Bioorg Med Chem 16: 7347-7357.

8. Alcon Research, Ltd. (2007) RNAi-mediated inhibition of spleen tyrosine-kinase related inflammatory conditions. WO/2007/121347.

9. Matsuda M, Park JG, Wang DC, Hunter S, Chien P, et al. (1996) Abrogation of the Fc gamma receptor IIA-mediated phagocytic signal by stem-loop Syk antisense oligonucleotides. Mol Biol Cell 7: 1095-1106.

10. Davis ME, Chen ZG, Shin DM (2008) Nanoparticle therapeutics: an emerging treatment modality for cancer. Nat Rev Drug Discov 7:771-782.

11. Cho K, Wang X, Nie S, Chen ZG, Shin DM. (2008) Therapeutic nanoparticles for drug delivery in cancer. Clin Cancer Res.14: 1310-1316.

12. Sinha R, Kim GJ, Nie S, Shin DM (2006) Nanotechnology in cancer therapeutics: bioconjugated nanoparticles for drug delivery. Mol Cancer Ther 5: 1909-17.

13. Uckun FM (2012) siRNA Carrying Targeted Nanoparticles as a New Class of Rationally Designed Anti-Cancer Therapeutics. J Nanomedic Biotherapeu Discover 2: e112.

14. Cely I, Yiv S, Yin Q, Shahidzadeh A, Tang L, et al (2012) Targeting Mantle Cell Lymphoma with Anti-SYK Nanoparticles. J Anal Oncolo 1: 1-8. 